\title{
A Note on a Core Catcher of a Cooperative Game
}

\author{
By T. S. H. Driessen ${ }^{1}$
}

\begin{abstract}
In Driessen (1986) it is shown that for games satisfying a certain condition the core of the game is included in the convex hull of the set of certain marginal worth vectors of the game, while it is conjectured that the inclusion holds without any condition on the game. In this note it is proved that the inclusion holds for all games.
\end{abstract}

Zusammenfassung: In Driessen (1986) wurde für Spiele, die eine gewisse Bedingung erfüllen, gezeigt, daß der Kern des Spieles in der konvexen Hülle von gewissen Vektoren der Marginalwerte liegt. Es wurde vermutet, daß diese Inklusion ohne weitere Bedingung an das Spiel gilt. In dieser Note wird nun gezeigt, daß die Inklusion für alle Spiele gilt.

Key words: cooperative game, core.

A cooperative $n$-person game in characteristic function form is a pair $(N, v)$ where $N=\{1,2, \ldots, n\}(n \geqslant 1)$ and $v$ is a real-valued function on the family $2^{N}$ of all subsets of $N$, satisfying $v(\emptyset)=0$. The function $v$ itself will also be called an $n$-person game. The family of all $n$-person games is denoted by $G^{n}$, while $\Theta^{n}$ denotes the set of all permutations on $N$.

Let $v \in G^{n}$. The core $C(v)$, the upper vector $b^{v}=\left(b_{1}^{v}, b_{2}^{v}, \ldots, b_{n}^{v}\right) \in \mathbb{R}^{n}$ and the gap function $g^{v}: 2^{N} \rightarrow \mathbb{R}$ of the game $v$ are defined by

$$
\begin{aligned}
& C(v):=\left\{x \in \mathbb{R}^{n} ; \sum_{j \in N} x_{j}=v(N) \text { and } \sum_{j \in S} x_{j} \geqslant v(S) \text { for all } S \subset N, S \neq \emptyset\right\} \\
& b_{i}^{v}:=v(N)-v(N-\{i\}) \quad \text { for all } i \in N, \quad g^{v}(\emptyset):=0 \text { and } \\
& g^{v}(S):=\sum_{j \in S} b_{j}^{v}-v(S) \quad \text { for all } S \subset N, S \neq \emptyset .
\end{aligned}
$$

In Driessen (1986; cf. proposition 2.1) it is shown that

$$
\begin{aligned}
& x_{i} \leqslant b_{i}^{v} \quad \text { for all } x \in C(v) \text { and all } i \in N \\
& g^{v}(N-\{i\})=g^{v}(N) \quad \text { for all } i \in N .
\end{aligned}
$$

1 T. S. H. Driessen, Department of Applied Mathematics, Twente University of Technology, Enschede, The Netherlands. 
Let $v \in G^{n}, k \in N$ and $\theta \in \Theta^{n}$. Then for a player $i \in N$ we define the set $P_{i}^{\theta}$ by $P_{i}^{\theta}:=$ $\{j \in N ; \theta(j)<\theta(i)\}$, while the marginal worth vector $x^{\theta, k}(v) \in \mathbb{R}^{n}$ of the game $v$ with respect to the coalition size up to $k$ is given by

$$
\begin{aligned}
x_{i}^{\theta, k}(v): & =v\left(P_{i}^{\theta} \cup\{i\}\right)-v\left(P_{i}^{\theta}\right) & & \text { if } \theta(i)<k \\
& =b_{i}^{v} & & \text { if } \theta(i)>k \\
& =b_{i}^{v}-g^{v}(N)+g^{v}\left(P_{i}^{\theta}\right) & & \text { if } \theta(i)=k .
\end{aligned}
$$

Note that $x_{i}^{\theta, n}(v)=v\left(P_{i}^{\theta} \cup\{i\}\right)-v\left(P_{i}^{\theta}\right)$ for all $i \in N$ because of (2). Let $W^{k}(v)$ be the convex hull of the set of all marginal worth vectors of the game $v$, i.e. $W^{k}(v):=$ conv $\left\{x^{\theta, k}(v) ; \theta \in \Theta^{n}\right\}$. For the interpretations of the above notions we refer to Driessen (1986), where it is also shown that the set $W^{k}(v)$ is a core catcher whenever a certain condition is satisfied by the game $v$.

Theorem 1 (cf. Theorem 5.2 in Driessen 1986): $C(v) \subset W^{k}(v)$ whenever $v \in G^{n}$ and $k \in N$ such that

$$
g^{v}(N) \geqslant g^{v}(S) \quad \text { for all } S \subset N \text { with }|S|=k-1 \text {. }
$$

Here $|S|$ denotes the number of elements of $S \subset N$. The purpose of this note is to prove that the inclusion $C(v) \subset W^{k}(v)$ holds for all $v \in G^{n}$ and all $k \in N$. The essential idea in the proof is to look at the corresponding game $v_{k} \in G^{n}$ defined by

$$
\begin{aligned}
v_{k}(S) & :=v(S) & & \text { if }|S|<k \\
& =v(N)-\sum_{j \in N-S} b_{j}^{v} & & \text { if }|S| \geqslant k .
\end{aligned}
$$

In the next lemma we give two properties of the game $v_{k}$ in relation to the original game $v$.

Lemma 2: Let $v \in G^{n}$ and $k \in N$. Then

(i) $C(v) \subset C\left(v_{k}\right)$

(ii) $x^{\theta, n}\left(v_{k}\right)=x^{\theta, k}(v)$ for all $\theta \in \Theta^{n}$.

Proof: (i) Let $x \in C(v)$. Then $\sum_{j \in N} x_{j}=v(N)=v_{k}(N), \sum_{j \in S} x_{j} \geqslant v(S)$ for all $S \neq \emptyset$, while for $S \subset N$ with $|S| \geqslant k$ we have also

$$
\sum_{j \in S} x_{j}=v(N)-\sum_{j \in N-S} x_{j} \geqslant v(N)-\sum_{j \in N-S} b_{j}^{v}=v_{k}(S)
$$

where the last inequality follows from (1). Hence, $x \in C\left(v_{k}\right)$.

(ii) Let $\theta \in \Theta^{n}$ and $i \in N$. If $\theta(i)<k$, then $\left|P_{i}^{\theta}\right|<k-1$ and hence

$$
x_{i}^{\theta, k}(v)=v\left(P_{i}^{\theta} \cup\{i\}\right)-v\left(P_{i}^{\theta}\right)=v_{k}\left(P_{i}^{\theta} \cup\{i\}\right)-v_{k}\left(P_{i}^{\theta}\right)=x_{i}^{\theta, n}\left(v_{k}\right) .
$$


If $\theta(i)>k$, then $\left|P_{i}^{\theta}\right| \geqslant k$ and hence

$$
x_{i}^{\theta, k}(v)=b_{i}^{v}=v_{k}\left(P_{i}^{\theta} \cup\{i\}\right)-v_{k}\left(P_{i}^{\theta}\right)=x_{i}^{\theta, n}\left(v_{k}\right)
$$

If $\theta(i)=k$, then $\left|P_{i}^{\theta}\right|=k-1$ and hence

$$
\begin{aligned}
x_{i}^{\theta, k}(v) & =b_{i}^{v}-g^{v}(N)+g^{v}\left(P_{i}^{\theta}\right)=v(N)-\sum_{j ; \theta(j)>k} b_{j}^{v}-v\left(P_{i}^{\theta}\right) \\
& =v_{k}\left(P_{i}^{\theta} \cup\{i\}\right)-v_{k}\left(P_{i}^{\theta}\right)=x_{i}^{\theta, n}\left(v_{k}\right) .
\end{aligned}
$$

We see that $x_{i}^{\theta, k}(v)=x_{i}^{\theta, n}\left(v_{k}\right)$ for all $i \in N$.

Now we are able to prove the fact that the set $W^{k}(v)$ is always a core catcher.

Theorem 3: $C(v) \subset W^{k}(v)$ for all $v \in G^{n}$ and all $k \in N$.

Proof: Let $v \in G^{n}$ and $k \in N$. By applying (2) with $v_{k}$ in the role of $v$, we have

$$
g^{v_{k}(N)}=g^{v_{k}(S)} \text { for all } S \subset N \text { with }|S|=n-1 \text {. }
$$

Now we conclude from Theorem 1 that $C\left(v_{k}\right) \subset W^{n}\left(v_{k}\right)$. By Lemma 2 we have also that $C(v) \subset C\left(v_{k}\right)$ and

$$
W^{n}\left(v_{k}\right)=\operatorname{conv}\left\{x^{\theta, n}\left(v_{k}\right) ; \theta \in \Theta^{n}\right\}=\operatorname{conv}\left\{x^{\theta, k}(v) ; \theta \in \Theta^{n}\right\}=W^{k}(v)
$$

Hence it follows that $C(v) \subset C\left(v_{k}\right) \subset W^{n}\left(v_{k}\right)=W^{k}(v)$.

We recall that the set $W^{k}(v)$ coincides with the core if and only if the game $v$ is a socalled $k$-convex game (cf. Theorem 5.3 in Driessen 1986). Finally we remark that the game $v_{k}$ as given by (3) turns out to be very useful to determine several solution concepts of $k$-convex games $v$ (Driessen 1985 and to appear).

\section{References}

Driessen TSH (1985) Contributions to the theory of cooperative games: the $\tau$-value and $k$-convex games. PhD Thesis, Catholic University, Nijmegen, The Netherlands

Driessen TSH (1986) $k$-Convex $n$-person games and their cores. Zeitschrift für Operations Research, Series A 30:49-64

Driessen TSH (1987) Solution concepts of $k$-convex $n$-person games. International Journal of Game Theory 16 (to appear) 